\title{
带非局部耗散项的守恒律方程大扰动解的整体 存在性
}

\author{
王利娟 ${ }^{1}$, 王维克 ${ }^{2 *}$, 徐金金 3
}

1. 上海对外经贸大学统计与信息学院, 上海 201620 ;

2. 上海交通大学数学科学学院, 上海 200240 ;

3. 北京应用物理与计算数学研究所, 北京 100084

E-mail: ljwang66@suibe.edu.cn, wkwang@sjtu.edu.cn, xuxinaboy@126.com

摘要 本文考虑带非局部耗散项的单个守恒律方程大扰动解的整体存在性. 首先, 针对方程次临界和 临界两种不同的情形, 利用 Green 函数方法和环形分解的技巧, 构造开放式高频估计方法, 得到了一 个新的解的正则性准则。然后, 利用极大值原理得到方程解的极大模的有界性, 验证了次临界情形下 解满足相应的正则性准则. 对于临界这一更困难的情形, 本文应用非线性极大值原理方法得到了更好 一点的有界性估计, 验证了临界情形下解也满足相应的正则性准则, 从而得到了 Cauchy 问题大扰动 经典解的整体存在性.

关键词 单个守恒律方程 非局部耗散项 Cauchy 问题 极大值原理 非线性极大值原理 MSC (2010) 主题分类 $35 \mathrm{~S} 05,35 \mathrm{~S} 10,35 \mathrm{~B} 40$

\section{1 引言}

\section{1 研究背景以及主要结论}

本文考虑带非局部耗散项的单个守恒律方程大扰动解的存在性, 方程的具体形式如下:

$$
\begin{cases}\partial_{t} u-\frac{\Delta}{(1-\Delta)^{\frac{s}{2}}} u=\operatorname{div} f(u), & x \in \mathbb{R}^{3}, \quad t>0, \\ u(0, x)=u_{0}(x), & x \in \mathbb{R}^{3},\end{cases}
$$

其中 $u=u(t, x):(0, \infty) \times \mathbb{R}^{3} \rightarrow \mathbb{R}$ 是未知函数, $u_{0}(x)$ 是初始值, $f(u)=\left(f_{1}(u), f_{2}(u), f_{3}(u)\right), f_{j}(u)=u^{2}$ $(j=1,2,3)$. 
方程 (1.1) 的非局部耗散项 $-\frac{\Delta}{(1-\Delta)^{\frac{5}{2}}} u$ 是利用拟微分算子来定义的:

$$
-\frac{\Delta}{(1-\Delta)^{\frac{s}{2}}} u=\mathcal{F}^{-1}\left\{\frac{|\xi|^{2}}{\left(1+|\xi|^{2}\right)^{\frac{s}{2}}} \mathcal{F} u\right\}
$$

为简洁起见, 在下文中, 记 $-\frac{\Delta}{(1-\Delta)^{\frac{s}{2}}} u=\Gamma_{s} u$.

对方程 (1.1) 的研究是基于下面两个方面的考虑: 一方面考虑的是, 如果在方程 (1.1) 中去掉非局 部耗散项, 方程 (1.1) 就是一个经典的守恒律方程. 其解即使对充分光滑的初始值, 也会出现解的 “爆 破” 现象 (参见文献 [1]), 但加上适当的耗散项后, 就可以抑制解的 “爆破”, 从而得到整体经典解的存 在性, 例如, 在方程 (1.1) 中, 当 $s=0$ 时, $\Gamma_{s}$ 就是一个经典的 Laplace 算子, 这时方程就是下面的带黏 性项的守恒律方程:

$$
\partial_{t} u+\nabla \cdot f(u)=\Delta u
$$

这时方程 (1.3) 的耗散项就可以有效地抑制解的 “爆破”, 得到经典解的整体存在性. 这方面有很多研 究工作. 例如, 在常状态附近大扰动解的整体存在性, 以及在一个黏性激波或者稀疏波附近扰动解的存 在性和稳定性等, 具体可参见文献 [2-8] 等.

当 $s=2$ 时, 方程 (1.1) 是形式如下的方程:

$$
\partial_{t} u+\nabla \cdot f(u)=\frac{\Delta}{1-\Delta} u
$$

这个模型一方面可以通过 Chapman-Enskog 展开从 Boltzmann 方程得出 (参见文献 [9]), 也可以看成 由下面的双曲 - 椭圆耦合方程组导出:

$$
\left\{\begin{array}{l}
\partial_{t} u+\operatorname{div} f(u)+\operatorname{div} q=0, \quad x \in \mathbb{R}^{3}, \quad t>0, \\
-\nabla \operatorname{div} q+q+\nabla u=0,
\end{array}\right.
$$

其中 $q \in \mathbb{R}^{3}$ 是辐射热通项. 这个方程是 3 维辐射气体运动模型的简化方程组. 如何从辐射气体运动 模型导出方程组 (1.5), 可参见文献 [10]. 在方程组 (1.5) 中, 可以看到 $\operatorname{div} q$ 可由 $-\frac{\Delta}{1-\Delta} u$ 表示, (1.5) 就 简化成 (1.4) 的形式. 对于方程 (1.4) 也有不少研究结果 (参见文献 [11-16]).

特别是在文献 [16] 中, 作者证明了对小扰动情形解是整体存在的, 但对于某些大扰动情形, 解是会 爆破的. 这就带来一个很有趣的问题, 对方程 (1.1), 当初值是某常状态的大扰动且 $s=0$ 时, 方程 (1.2) 的解是整体存在的; 但当 $s=2$ 时对应的方程 (1.4), 其解会出现 “爆破” 的现象. 那么,一个很自然的 问题是, 是否存在一个 $s^{*}$, 当 $s<s^{*}$ 时解是整体存在的; 当 $s>s^{*}$ 时解会爆破; 特别地, 当 $s=s^{*}$ 时, 这个临界情形下解的情形更是人们关心的问题.

本文的另外一个重要的研究动机与 surface quasi-geostrophic (SQG) 方程的研究有关, SQG 方程 的形式如下:

$$
\begin{cases}\partial_{t} \theta+u \cdot \nabla \theta+\kappa \Lambda^{\mu} \theta=0, & x \in \mathbb{R}^{2}, \quad t>0, \\ u=\mathcal{R}^{\perp} \theta=\left(-\mathcal{R}_{2} \theta, \mathcal{R}_{1} \theta\right), & x \in \mathbb{R}^{2},\end{cases}
$$

其中 $\Lambda=\sqrt{-\Delta}, \mathcal{R}_{j}$ 是 Riesz 变换, $\kappa$ 是一个常数.

关于 SQG 的物理背景可参见文献 [17,18]. 对这个方程 Cauchy 问题大扰动解的整体存在性的研 究有着大量重要的工作. 当 $\mu>1$ 时 (通常称为次临界情形) 已经证明方程整体解是存在的 (参见文 
献 [19-21]). 当 $\mu=1$, 即临界情形, 在数学上是颇具挑战性的问题. 在文献 [22-25] 中, 几位作者用完 全不同的方法证明整体解的存在性, 均发表在重要的学术杂志上. 这个现象表明, 人们在这一问题研 究中不仅仅看重研究结果, 更看重的是在这个方程研究中所使用的一些独特方法. 例如, Kiselev 等 ${ }^{[25]}$ 引入连续模的方法得到临界情形下带耗散结构的 SQG 方程的大扰动周期解的整体存在性; Caffarelli 等 ${ }^{[22]}$ 利用调和扩张及 De Giorgi-Nash-Moser 迭代技术给出了整体解的存在性和正则性结果; Constantin 等 $[23,24]$ 引入的非线性极大值原理的方法, 又一次讨论了临界情形下带耗散结构的 SQG 方程 的大扰动解整体存在性, 使人们更清楚地看到非局部耗散机制在解的存在性上如何起作用, 等等.

还应提到与 SQG 方程十分类似的带分数阶黏性项的 Burgers 方程

$$
\partial_{t} v+\sum_{j=1}^{n} v \partial_{x_{j}} v+\kappa \Lambda^{\mu} v=0, \quad x \in \mathbb{R}^{n}, \quad t>0 .
$$

当 $\mu \geqslant 1$ 时, 即对于次临界和临界情形, 有一系列和 SQG 方程类似与平行的结果 (参见文献 $[24,26,27]$ ). 对超临界的情形, 文献 $[28,29]$ 对一维情形已经证明 $\mu<1$ 时解会爆破. 与 (1.7) 中的耗散项 $\Lambda^{\mu} u$ 比 较, 方程 (1.1) 中的非局部的耗散项 $\Gamma_{s} u$ 有类似和不同之处. 类似之处在于对 $\Lambda^{\mu}$ 和 $\Gamma_{s}$ 的 Fourier 变 换分别为 $|\xi|^{\mu}$ 和 $\frac{|\xi|^{2}}{\left(1+|\xi|^{2}\right)^{s / 2}}$, 当 $|\xi|$ 充分大时, $\frac{|\xi|^{2}}{\left(1+|\xi|^{2}\right)^{s / 2}}$ 等价于 $|\xi|^{2-s}$, 取 $\mu=2-s$, 情况是一致的. 但 写出这两个算子在空间维数 $n$ 的情形下的核形式 (参见文献 $[24,30]$ ) 分别为

$$
\Lambda^{\mu} u(x)=C_{\mu} \mathrm{P} . \mathrm{V} \cdot \int_{\mathbb{R}^{n}} \frac{u(x)-u(y)}{|x-y|^{n+\mu}} d y
$$

和

$$
\Gamma_{s} u(x)=\frac{\Delta}{(1-\Delta)^{\frac{s}{2}}} u(x)=\mathcal{K}_{s}(\cdot) * \Delta u(\cdot, t)
$$

其中 $C_{\mu}>0$, 并且

$$
\mathcal{K}_{s}(x)=(4 \pi)^{-\frac{s}{2}} \frac{1}{\Gamma(s / 2)} \int_{0}^{\infty} \mathrm{e}^{-\pi|x|^{2} / \delta} \mathrm{e}^{-\delta / 4 \pi} \delta^{(-n+s) / 2} \frac{d \delta}{\delta} .
$$

可以看到对算子 $\Lambda^{\mu}$, 其核有正性 (见正性引理, 命题 3.4), 但 $\Gamma_{s}$ 的核不具有正性. 这正是本文在证明 其解的整体存在性时所遇到的困难之一.

本文主要考虑方程 (1.1) 在次临界和临界情形下 (即 $0 \leqslant s<1$ 和 $s=1$ ), 其 Cauchy 问题大扰动 解的适定性. 首先, 利用极大值原理 (定理 3.3) 得到方程解的 $L^{p}(p \in[2,+\infty])$ 的有界性, 然后希望通 过提高方程解的正则性, 得到方程经典解的整体存在性. 本文利用环形分解和 Green 函数的方法得到 了一个新的正则性准则 (定理 2.1) 来提高方程解的正则性. 其主要想法是借助于 Green 函数和开放式 高频估计方法来得到这一正则性准则. 这样, 提高方程解的正则性就只需要证明解满足这一新的正则 性准则. 对于次临界的情形, 满足正则性准则仅需要解是 $L^{\infty}$ 有界, 这由极大值原理就可以得到. 但对 于临界的情形, 为了满足正则性准则, 我们还需要先借助于非线性极大值原理得到方程解 $u(t, x) \in C^{\alpha}$ $(0<\alpha \ll 1)$.

本文的主要结论如下:

定理 1.1 当 $0 \leqslant s \leqslant 1$ 时, 初值满足 $u_{0} \in L^{1} \cap C^{\gamma}\left(\mathbb{R}^{3}\right)$, 则 Cauchy 问题 (1.1) 存在唯一的经典 解 $u(t, x)$, 而且满足

$$
u \in L^{\infty}\left([0, \infty) ; C^{\gamma}\left(\mathbb{R}^{3}\right)\right), \quad \gamma>1
$$




\section{2 符号和本文的安排}

下面列举本文中即将用到的数学符号. $\hat{f}(t, \xi)$ 表示 Fourier 变换, 定义如下:

$$
\hat{f}(t, \xi)=(\mathcal{F} f)(t, \xi)=\int_{\mathbb{R}^{3}} f(t, x) \mathrm{e}^{-\mathrm{i} x \cdot \xi} d x .
$$

Fourier 逆变换为

$$
f(t, x)=(2 \pi)^{-3} \int_{\mathbb{R}^{3}} \hat{f}(t, \xi) \mathrm{e}^{\mathrm{i} x \cdot \xi} d \xi .
$$

记 $\Lambda=(-\Delta)^{\frac{1}{2}}, \alpha=\left(\alpha_{1}, \alpha_{2}, \alpha_{3}\right),|\alpha|=\sum_{i=1}^{3} \alpha_{i}, D^{\alpha}=D_{x_{1}}^{\alpha_{1}} D_{x_{2}}^{\alpha_{2}} D_{x_{3}}^{\alpha_{3}}$. 对于非负实数 $m, H^{m}\left(\mathbb{R}^{3}\right)$ 为 以 $\|\cdot\|_{H^{m}}$ 为范数的 Sobolev 空间.

本文的具体安排如下: 第 2 节建立方程 (1.1) 解的正则性准则, 用来提高方程解的正则性. 值得指 出的是, 正则性准则的关键是需要得到当 $0 \leqslant s<1$ 时, 有 $\|u\|_{L^{\infty}} \leqslant C$; 当 $s=1$ 时, 有 $\|u\|_{C^{\alpha}} \leqslant C$. 为 此, 第 3 节利用极大值原理给出了当 $0 \leqslant s \leqslant 1$ 时方程的解 $u(t, x)$ 在 $L^{p}$ 范数下的有界性. 第 4 节利 用非线性极大值原理的方法得到了 $s=1$ 情形下方程的解 $u(t, x)$ 在 $C^{\alpha}(\alpha \ll 1)$ 范数下的有界性. 最 后在附录给出了第 2 节中所需要的 Green 函数的逐点估计.

\section{2 正则性准则}

为了提高方程 (1.1) 解的正则性, 我们利用 Green 函数结合开放式高频估计的方法建立了方程解 的正则性准则.

定理 2.1 设初值 $u_{0}(x)$ 满足 $u_{0}(x) \in L^{1}\left(\mathbb{R}^{3}\right) \cap C^{\gamma}\left(\mathbb{R}^{3}\right)(\gamma>1), u(t, x)$ 是方程 $(1.1)$ 的解且满足

(1) 当 $0 \leqslant s<1$ 时, 有

$$
u(t, x) \in L^{\infty}\left([0,+\infty), L^{1}\left(\mathbb{R}^{3}\right) \cap L^{\infty}\left(\mathbb{R}^{3}\right)\right) ;
$$

(2) 当 $s=1$ 时, 假设存在正数 $\alpha>0$, 使得

$$
u(t, x) \in L^{\infty}\left([0,+\infty), L^{1}\left(\mathbb{R}^{3}\right) \cap C^{\alpha}\left(\mathbb{R}^{3}\right)\right),
$$

那么有 $u(t, x) \in L^{\infty}\left([0,+\infty), C^{\gamma}\left(\mathbb{R}^{3}\right)\right)$.

为了证明上面的定理, 我们引进以下标准的环形分解的记号. 记 $\mathcal{C}=\left\{\xi \in \mathbb{R}^{3}\left|\frac{5}{6}<\right| \xi \mid<\frac{12}{5}\right\}$ 为 环形, $\mathcal{B}=\left\{\xi \in \mathbb{R}^{3}|0 \leqslant| \xi \mid<\frac{6}{5}\right\}$ 为球, 则存在两个值域为 $[0,1]$ 的函数 $\psi$ 和 $\varphi$, 并且 $\psi \in C_{0}^{\infty}(\mathcal{B})$, $\varphi \in C_{0}^{\infty}(\mathcal{C})$, 使得

$$
\forall \xi \in \mathbb{R}^{3}, \quad \psi(\xi)+\sum_{j \geqslant 0} \varphi\left(2^{-j} \xi\right)=1 .
$$

容易验证,

$$
\begin{aligned}
& \left|j-j^{\prime}\right| \geqslant 2 \Rightarrow \operatorname{supp} \varphi\left(2^{-j} \cdot\right) \cap \operatorname{supp} \varphi\left(2^{-j^{\prime}} \cdot\right)=\emptyset, \\
& j \geqslant 1 \Rightarrow \operatorname{supp} \psi \cap \operatorname{supp} \varphi\left(2^{-j} \cdot\right)=\emptyset,
\end{aligned}
$$

其中 $\Delta_{j}$ 定义如下:

$$
\Delta_{j} u=\varphi\left(2^{-j} D\right) u=: \mathcal{F}^{-1}\left(\varphi\left(2^{-j} \cdot\right) \hat{u}(\cdot)\right), \quad j \geqslant 0,
$$




$$
\Delta_{-1} u=\psi(D) u=: \mathcal{F}^{-1}(\psi(\cdot) \hat{u}(\cdot))
$$

非齐次的低频截断算子 $S_{q}(D)$ 定义为

$$
S_{q}(D)=\psi(D)+\sum_{0 \leqslant j \leqslant q-2} \varphi\left(2^{-j} D\right)=\sum_{-1 \leqslant j \leqslant q-2} \Delta_{j},
$$

高频的截断算子 $T_{q}(D)$ 定义为

$$
T_{q}(D)=\operatorname{Id}-S_{q}(D),
$$

其中 Id 是恒等算子. 由上面的定义很容易得到下面的分解:

$$
\mathrm{Id}=S_{q}(D)+T_{q}(D)
$$

根据 Hölder 空间 $C^{\gamma}\left(\mathbb{R}^{3}\right)$ 的定义, 范数 $\|u\|_{C^{\gamma}}$ 定义如下:

(1) 当 $0<\gamma<1$ 时,

$$
\|u\|_{C^{\gamma}}=\|u\|_{L^{\infty}}+[u]_{\gamma},
$$

其中,

$$
[u]_{\gamma}=\sup _{\substack{(x, y) \in \mathbb{R}^{3} \times \mathbb{R}^{3}, x \neq y .}} \frac{|u(x)-u(y)|}{|x-y|^{\gamma}}<\infty .
$$

(2) 当 $\gamma>1$ 时, 令 $[\gamma]$ 为 $\gamma$ 的取整函数, $\rho=\gamma-[\gamma]$, 那么,

$$
\|u\|_{C^{\gamma}}=\sum_{|\lambda| \leqslant[\gamma]}\left\|D^{\lambda} u\right\|_{L^{\infty}}+\sum_{|\lambda|=[\gamma]}\left[D^{\lambda} u\right]_{\rho} .
$$

对于环形分解, 我们有下列的引理:

引理 2.2 假设 $\gamma \in \mathbb{R}^{+} \backslash \mathbb{N}$, 则下列 4 个结论是等价的:

(1) $u \in C^{\gamma}\left(\mathbb{R}^{3}\right)$;

(2) 对于 $u=\sum_{p \geqslant-1} \Delta_{p} u$ 和任意的 $p \geqslant-1$, 有 $\left\|\Delta_{p} u\right\|_{L^{\infty}} \leqslant C \cdot 2^{-p \gamma}$;

(3) 对于 $u=\sum_{p \geqslant-1} \Delta_{p} u$, 存在正整数 $P_{0}$, 使得当 $p>P_{0}$ 时,

$$
\left\|\Delta_{p} u\right\|_{L^{\infty}} \leqslant C \cdot 2^{-p \gamma}
$$

(4) 对于 $u=\sum_{p \geqslant-1} \Delta_{p} u$, 存在正整数 $P_{0}$, 使得当 $p>P_{0}$ 时,

$$
\left\|T_{p} u\right\|_{L^{\infty}} \leqslant C \cdot 2^{-p \gamma} .
$$

证明 关于 (1) 和 (2) 的等价性的结果是经典的, 文献 [31] 给出了详细的证明. 由级数收玫的定 义知, (2)-(4) 的等价性是显然的.

接下来将给出定理 2.1 的证明. 在此之前, 先给出方程局部解的存在性定理.

定理 2.3 (局部存在性) 假设初值满足 $u_{0}(x) \in C^{\gamma}\left(\mathbb{R}^{3}\right), \gamma>1$, 则存在依赖于 $\left\|u_{0}\right\|_{C^{\gamma}}$ 的正常数 $\delta_{0}$, 使得当 $t<2 \delta_{0}$ 时, 方程 (1.1) 存在局部解 $u(t, x)$, 而且满足

$$
u(t, x) \in L^{\infty}\left(\left[0,2 \delta_{0}\right] ; C^{\gamma}\left(\mathbb{R}^{3}\right)\right) .
$$


关于这类方程局部解的存在性的证明有很多经典的文章, 如文献 [32], 为避免繁冗, 具体的证明过 程这里省略.

由引理 2.2 可知, 要证明定理 2.1 , 只需要证明方程的解 $u(t, x)$ 在 $t>\delta_{0}$ 时满足 $(2.5)$. 本文将利 用 Green 函数的方法来证明方程 Cauchy 问题 (1.1) 的解 $u(t, x)$ 满足条件 (2.5).

Green 函数 $G=G(t, x)$ 是 Cauchy 问题 (1.1) 的基本解, 也即为下述线性方程的解:

$$
\begin{cases}\partial_{t} G-\frac{\Delta}{(1-\Delta)^{\frac{s}{2}}} G=0, & x \in \mathbb{R}^{3}, \quad t>0, \\ \left.G\right|_{t=0}=\delta(x), & x \in \mathbb{R}^{3},\end{cases}
$$

其中 $\delta(x)$ 是 Dirac 函数. 显然, 我们可以很轻松地得到 Green 函数 $G(t, \xi)$ 的 Fourier 变换下的表达式:

$$
\widehat{G}(t, \xi)=\exp \left(-\frac{|\xi|^{2} t}{\left(1+|\xi|^{2}\right)^{s / 2}}\right) .
$$

关于 Green 函数 $G(t, x)$ 的估计, 我们有下面的定理:

定理 2.4 对于任意的复指标 $\alpha=\left(\alpha_{1}, \alpha_{2}, \alpha_{3}\right)$, Green 函数 $G(t, x)$ 有下面的衰减性:

(1) 当 $0<t<1$ 时,

$$
\left\|D^{\alpha} G(t)\right\|_{L^{p}} \leqslant C t^{-\frac{|\alpha|}{2-s}-\frac{3}{2-s}\left(1-\frac{1}{p}\right)}, \quad 1 \leqslant p \leqslant \infty
$$

(2) 当 $t \geqslant 1$ 时,

$$
\left\|D^{\alpha} G(t)\right\|_{L^{p}} \leqslant C(1+t)^{-\frac{|\alpha|}{2}-\frac{3}{2}\left(1-\frac{1}{p}\right)}, \quad 1 \leqslant p \leqslant \infty .
$$

当 $p \geqslant 2$ 时, 利用 Young 不等式及 $\hat{G}(t, \xi)$ 的表达式, 很容易地得到 $\left\|D^{\alpha} G(t)\right\|_{L^{p}}$ 的衰减估计. 然 而, 当 $1 \leqslant p<2$ 时, 由于不能再利用 Young 不等式, 因此, 其证明不是显然的. 我们的办法是首先得 到 Green 函数的逐点估计, 然后利用其逐点估计来得到方程的解 $\left\|D^{\alpha} G(t)\right\|_{L^{p}}$ 的衰减. 关于 Green 函 数的逐点估计的证明, 参见文献 [33,34]. 为方便读者并避免繁冗, 我们把 Green 函数逐点估计的证明 放在附录.

利用 Green 函数容易得到方程 (1.1) 解的线性部分的估计. 接下来, 其关键部分是非线性部分的 估计. 下面的引理是开放式高频估计方法的关键性引理:

引理 2.5 假设初值满足 $u_{0}(x) \in L^{1}\left(\mathbb{R}^{3}\right) \cap C^{\gamma}\left(\mathbb{R}^{3}\right)(\gamma>1), u(t, x)$ 是方程 $(1.1)$ 的解, 则有

(1) 当 $0 \leqslant s<1$ 时, 如果

$$
u(t, x) \in L^{\infty}\left([0,+\infty), L^{1}\left(\mathbb{R}^{3}\right) \cap L^{\infty}\left(\mathbb{R}^{3}\right)\right),
$$

那么,

$$
\left\|T_{q} u^{2}\right\|_{L^{2}}+\left\|T_{q} u^{2}\right\|_{L^{\infty}} \leqslant C\left\|T_{q-3} u\right\|_{L^{\infty}}
$$

(2) 当 $s=1$ 时, 如果

$$
u(t, x) \in L^{\infty}\left([0,+\infty), L^{1}\left(\mathbb{R}^{3}\right) \cap C^{\alpha}\left(\mathbb{R}^{3}\right)\right), \quad \alpha>0,
$$

那么,

$$
\left\|\Lambda^{\beta} T_{q} u^{2}\right\|_{L^{\infty}} \leqslant C\left\|T_{q-3} u\right\|_{L^{\infty}}, \quad \beta=\frac{\alpha}{2} .
$$


证明 (1) 令 $A_{q}=\left\|T_{q} u\right\|_{L^{\infty}}$, 当 $0 \leqslant s<1$ 时, 根据 $T_{q}$ 的定义和 $T_{q}\left(\left(S_{q-3} u\right)^{2}\right)=0$, 可以得到

$$
\begin{aligned}
\left\|T_{q} u^{2}\right\|_{L^{\infty}} & =\left\|T_{q}\left(T_{q-3} u+S_{q-3} u\right)^{2}\right\|_{L^{\infty}} \\
& \leqslant C\left\|T_{q}\left(T_{q-3} u\right)^{2}\right\|_{L^{\infty}}+2\left\|T_{q}\left(T_{q-3} u\right)\left(\left(\mathrm{Id}-T_{q-3}\right) u\right)\right\|_{L^{\infty}} \\
& \leqslant C A_{q-3}+2\left\|\left(\mathrm{Id}-T_{q-3}\right) u\right\|_{L^{\infty}}\left\|T_{q-3} u\right\|_{L^{\infty}} \\
& \leqslant C A_{q-3},
\end{aligned}
$$

其中, 我们利用了 $\|u\|_{L^{\infty}} \leqslant C$ 这个假设. 同理可以得到 $\left\|T_{q} u^{2}\right\|_{L^{2}} \leqslant C A_{q-3}$.

(2) 假设 $u(t, x) \in L^{\infty}\left([0,+\infty), L^{1}\left(\mathbb{R}^{3}\right) \cap C^{\alpha}\left(\mathbb{R}^{3}\right)\right)$. 分数阶的 Laplace 算子的计算公式为 (参见文 献 [35])

$$
\Lambda^{\beta} u(x)=C_{\beta} \mathrm{P} . \mathrm{V} \cdot \int \frac{u(x)-u(y)}{|x-y|^{3+\beta}} d y
$$

其中 $C_{\beta}>0$. 于是,

$$
\begin{aligned}
\Lambda^{\beta}\left(T_{q} u^{2}\right) & =C_{\beta} \mathrm{P} . \mathrm{V} \cdot \int_{\mathbb{R}^{3}} \frac{\left(T_{q} u^{2}\right)(t, x)-\left(T_{q} u^{2}\right)(t, y)}{|x-y|^{3+\beta}} d y \\
& =C_{\beta} \mathrm{P} . \mathrm{V} \cdot\left(\int_{|x-y| \leqslant 1}+\int_{|x-y|>1}\right) \frac{\left(T_{q} u^{2}\right)(t, x)-\left(T_{q} u^{2}\right)(t, y)}{|x-y|^{3+\beta}} d y \\
& =: E_{1}+E_{2} .
\end{aligned}
$$

接下来将用不同的方法来处理这两个不同的项 $E_{1}$ 和 $E_{2}$. 对于 $E_{1}$ 来说, 不失一般性, 假设存在点 $\left(t_{0}, x_{0}\right)$, 使得 $u\left(t_{0}, x_{0}\right)=0$. 否则, 我们可以利用 $u\left(t, x-u\left(t_{0}, x_{0}\right)\left(t-t_{0}\right)\right)-u\left(t_{0}, x_{0}\right)$ 来代替 $u(t, x)$. 由 于 $T_{q}\left(\left(S_{q-3} u\right)^{2}\right)=0$, 那么,

$$
\begin{aligned}
\left|\left(T_{q} u^{2}\right)\left(t_{0}, y\right)-\left(T_{q} u^{2}\right)\left(t_{0}, x_{0}\right)\right|= & \mid T_{q}\left(T_{q-3} u\right)^{2}\left(t_{0}, y\right)+2 T_{q}\left[\left(T_{q-3} u\right)\left(S_{q-3} u\right)\right]\left(t_{0}, y\right)-T_{q}\left(T_{q-3} u\right)^{2}\left(t_{0}, x_{0}\right) \\
& -2 T_{q}\left[\left(T_{q-3} u\right)\left(S_{q-3} u\right)\right]\left(t_{0}, x_{0}\right) \mid .
\end{aligned}
$$

注意到我们的假设 $u\left(t_{0}, x_{0}\right)=0$, 则

$$
\left|\left(T_{q-3} u\right)\left(t_{0}, y\right)-\left(T_{q-3} u\right)\left(t_{0}, x_{0}\right)\right| \leqslant C\left|y-x_{0}\right|^{2 \beta}, \quad\left|u\left(t_{0}, y\right)-u\left(t_{0}, x_{0}\right)\right| \leqslant C\left|y-x_{0}\right|^{2 \beta},
$$

所以有

$$
\begin{aligned}
\left|\left(T_{q} u^{2}\right)\left(t_{0}, y\right)-\left(T_{q} u^{2}\right)\left(t_{0}, x_{0}\right)\right| \leqslant & \left|\left[\left(T_{q-3} u\right)\left(t_{0}, y\right)\right]^{2}-\left[\left(T_{q-3} u\right)\left(t_{0}, x_{0}\right)\right]^{2}\right| \\
& +2\left|\left(T_{q-3} u\right)\left(t_{0}, y\right)\left(u\left(t_{0}, y\right)-u\left(t_{0}, x_{0}\right)\right)\right| \\
\leqslant & \left|\left[\left(T_{q-3} u\right)\left(t_{0}, y\right)+\left(T_{q-3} u\right)\left(t_{0}, x_{0}\right)\right]\left[\left(T_{q-3} u\right)\left(t_{0}, y\right)-\left(T_{q-3} u\right)\left(t_{0}, x_{0}\right)\right]\right| \\
& +2\left|\left(T_{q-3} u\right)\left(t_{0}, y\right)\left(u\left(t_{0}, y\right)-u\left(t_{0}, x_{0}\right)\right)\right| \\
\leqslant & C A_{q-3}\left|y-x_{0}\right|^{2 \beta} .
\end{aligned}
$$

因此,

$$
\begin{aligned}
\left|E_{1}\left(t_{0}, x_{0}\right)\right| & \leqslant C_{\beta} \text { P.V. } \int_{\left|x_{0}-y\right| \leqslant 1}\left|\frac{T_{q} u^{2}\left(t_{0}, x_{0}\right)-T_{q} u^{2}\left(t_{0}, y\right)}{\left|x_{0}-y\right|^{3+\beta}}\right| d y \\
& \leqslant C A_{q-3} \int_{\left|y-x_{0}\right| \leqslant 1} \frac{1}{\left|y-x_{0}\right|^{3-\beta}} d y \leqslant C A_{q-3},
\end{aligned}
$$


其中 $C$ 是一个与 $\left(t_{0}, x_{0}\right)$ 无关的常数. 所以,

$$
\left\|E_{1}\right\|_{L^{\infty}} \leqslant C A_{q-3} .
$$

对于 $E_{2}$ 而言, 我们很容易得到下面的估计:

$$
\left\|E_{2}\right\|_{L^{\infty}} \leqslant C\left\|\int_{|x-y|>1} \frac{T_{q} u^{2}(t, x)-T_{q} u^{2}(t, y)}{|x-y|^{3+\beta}} d y\right\|_{L^{\infty}} \leqslant C A_{q-3} .
$$

将 $E_{1}$ 和 $E_{2}$ 的估计结合在一起, 则可以得到

$$
\left\|\Lambda^{\beta} T_{q} u^{2}\right\|_{L^{\infty}} \leqslant C A_{q-3} .
$$

证毕.

定理 2.1 的证明 要证明方程 (1.1) 的解当 $t>\delta_{0}$ 时满足 (2.5). 根据 Duhamel 原理知, 方程 (1.1) 的解可以表示为

$$
u(t, x)=G(t, \cdot) * u_{0}+\int_{0}^{t} G(t-\tau) * \operatorname{div} f(u) d \tau .
$$

根据 $u(t, x)$ 的表示形式, 可以得到

$$
T_{q} u=T_{q} G(t, \cdot) * u_{0}+\int_{0}^{t} T_{q} G(t-\tau) * \operatorname{div} f(u) d \tau=: \mathrm{I}_{q}+\mathrm{II}_{q} .
$$

关于 $\mathrm{I}_{q}$ 的估计, 当 $t>\delta_{0}$ 时, 利用 Young 不等式, 有

$$
\begin{aligned}
\left\|\mathrm{I}_{q}\right\|_{L^{\infty}} & =\left\|T_{q} G(t) * u_{0}\right\|_{L^{\infty}} \leqslant\left\|T_{q} G(t)\right\|_{L^{2}}\left\|u_{0}\right\|_{L^{2}} \\
& \leqslant\left(\int_{\mathbb{R}^{3}}\left(\widehat{T}_{q}(\xi) \mathrm{e}^{-\frac{|\xi|^{2} t}{\left(1+|\xi|^{2}\right)^{s / 2}}}\right)^{2} d \xi\right)^{1 / 2}\left\|u_{0}\right\|_{L^{2}} \\
& \leqslant C \mathrm{e}^{-2^{q(2-s)} \delta_{0}} .
\end{aligned}
$$

通过直接计算, 我们可以总结为存在正数 $Q_{1}$, 使得对于任意 $q>Q_{1}$, 有

$$
\left\|\mathrm{I}_{q}\right\|_{L^{\infty}} \leqslant C \cdot 2^{-q \gamma} .
$$

接下来将要估计 $\left\|\mathrm{II}_{q}\right\|_{L^{\infty}}$. 首先将 $\mathrm{II}_{q}$ 分解为两部分,

$$
\mathrm{II}_{q}=\int_{0}^{t-\varepsilon} T_{q} G(t-\tau) * \operatorname{div} f(u) d \tau+\int_{t-\varepsilon}^{t} T_{q} G(t-\tau) * \operatorname{div} f(u) d \tau=: \mathrm{II}_{q}^{1}+\mathrm{II}_{q}^{2},
$$

其中 $\varepsilon$ 为一个待定常数. 对于 $\mathrm{II}_{q}^{1}$, 有

$$
\begin{aligned}
\left\|\mathrm{II}_{q}^{1}\right\|_{L^{\infty}} & =\left\|\int_{0}^{t-\varepsilon} T_{q} G(t-\tau) * \operatorname{div} f(u) d \tau\right\|_{L^{\infty}} \\
& \leqslant \int_{0}^{t-\varepsilon}\left\|T_{q} \Lambda G(t-\tau)\right\|_{L^{2}}\left\|T_{q} f(u)\right\|_{L^{2}} d \tau \\
& \leqslant \mathrm{e}^{-2^{q(2-s)} \varepsilon} \int_{0}^{t-\varepsilon}\left\|\widehat{T}_{q}(\xi)|\xi| \mathrm{e}^{-\frac{|\xi|^{2}(t-\tau)}{2\left(1+|\xi|^{2}\right)^{s / 2}}}\right\|_{L^{2}}\left\|T_{q}\left(u^{2}\right)\right\|_{L^{2}} d \tau .
\end{aligned}
$$


根据 (2.8), 可得

$$
\left\|\mathrm{II}_{q}^{1}\right\|_{L^{\infty}} \leqslant C A_{q-3} \mathrm{e}^{-2^{q(2-s)}} \varepsilon \varepsilon^{-\frac{1}{(2-s)}} \leqslant C A_{q-3} \mathrm{e}^{-2^{q}\left(\frac{\varepsilon}{2}\right)} .
$$

为了估计 $\mathrm{II}_{q}^{2}$, 我们分成 $0 \leqslant s<1$ 和 $s=1$ 两种情形来进行证明. 首先, 当 $0 \leqslant s<1$ 时, 利用 (2.6) 和 (2.8), 得到

$$
\begin{aligned}
\left\|\mathrm{II}_{q}^{2}\right\|_{L^{\infty}} & =\left\|\int_{t-\varepsilon}^{t} T_{q} G(t-\tau) * \operatorname{div} f(u) d \tau\right\|_{L^{\infty}} \\
& \leqslant \int_{t-\varepsilon}^{t}\left\|\Lambda T_{q} G(t-\tau)\right\|_{L^{1}}\left\|T_{q}\left(u^{2}\right)\right\|_{L^{\infty}} d \tau \\
& \leqslant C A_{q-2} \int_{t-\varepsilon}^{t}(t-\tau)^{-\frac{1}{2-s}} d \tau \leqslant C A_{q-2} \varepsilon^{(1-s) /(2-s)} .
\end{aligned}
$$

对于 $s=1$, 利用 $(2.6)$ 和 $(2.9)$, 得到

$$
\begin{aligned}
\left\|\mathrm{II}_{q}^{2}\right\|_{L^{\infty}} & \leqslant \int_{t-\varepsilon}^{t}\left\|T_{q} \Lambda^{1-\beta} G(t-\tau)\right\|_{L^{1}}\left\|\Lambda^{\beta} T_{q}\left(u^{2}\right)\right\|_{L^{\infty}} d \tau \\
& \leqslant C A_{q-3} \int_{t-\varepsilon}^{t}(t-\tau)^{-(1-\beta)} d \tau \leqslant C A_{q-3} \varepsilon^{\beta} .
\end{aligned}
$$

令

$$
\tilde{\varepsilon}_{0}= \begin{cases}C \varepsilon^{\frac{1-s}{2-s}}, & 0 \leqslant s<1 \\ C \varepsilon^{\beta}, & s=1\end{cases}
$$

将 (2.10)-(2.16) 加起来, 可得

$$
A_{q}=\left\|T_{q} u\right\|_{L^{\infty}} \leqslant\left(\tilde{\varepsilon}_{0}+\mathrm{e}^{-2^{q}\left(\frac{\varepsilon}{2}\right)}\right) A_{q-3}+C \cdot 2^{-\gamma q} .
$$

接下来要证明对于任意的正数 $l$, 都有

$$
\left(\mathrm{H}_{l}\right): \quad A_{q+3 l}=\left\|T_{q+3 l} u\right\|_{L^{\infty}} \leqslant\left(\tilde{\varepsilon}_{0}+\mathrm{e}^{-2^{q}\left(\frac{\varepsilon}{2}\right)}\right)^{l} A_{q}+C \cdot 2^{-\gamma(q+3 l)} .
$$

我们将利用数学归纳法来进行证明上述结论.

由 (2.17) 很显然可以得到当 $l=1$ 时 (2.18) 成立. 接下来假设当 $k=l-1$ 时 (2.18) 成立, 即

$$
A_{q+3(l-1)}=\left\|T_{q+3(l-1)} u\right\|_{L^{\infty}} \leqslant\left(\tilde{\varepsilon}_{0}+\mathrm{e}^{-2^{q}\left(\frac{\varepsilon}{2}\right)}\right)^{l-1} A_{q}+C \cdot 2^{-\gamma(q+3(l-1))} .
$$

又根据 Duhamel 原理, 由 $\mathrm{I}_{q+3 l}$ 的表达形式以及 Green 函数的性质, 有

$$
\left\|\mathrm{I}_{q+3 l}\right\|_{L^{\infty}} \leqslant C \cdot 2^{-\gamma(q+3 l)} .
$$

类似于 (2.17) 的证明过程, 我们有

$$
\begin{aligned}
\left\|T_{q+3 l} u\right\|_{L^{\infty}} & \leqslant\left(\tilde{\varepsilon}_{0}+\mathrm{e}^{-2^{q}\left(\frac{\varepsilon}{2}\right)}\right) A_{q+3(l-1)}+C \cdot 2^{-\gamma(q+3 l)} \\
& \leqslant\left(\tilde{\varepsilon}_{0}+\mathrm{e}^{-2^{q}\left(\frac{\varepsilon}{2}\right)}\right)^{l} A_{q}+C \cdot\left[\left(\tilde{\varepsilon}_{0}+\mathrm{e}^{-2^{q}\left(\frac{\varepsilon}{2}\right)}\right) 2^{3 \gamma}+1\right] 2^{-\gamma(q+3 l)} \\
& =\left(\tilde{\varepsilon}_{0}+\mathrm{e}^{-2^{q}\left(\frac{\varepsilon}{2}\right)}\right)^{l} A_{q}+C \cdot 2^{-\gamma(q+3 l)},
\end{aligned}
$$


即 (2.18) 对于 $l$ 仍然是成立的. 所以, 根据数学归纳法, 我们得到 (2.18) 对于任意的正数 $l$ 都成立.

最后, 令 $\tilde{\varepsilon}_{0}=2^{-6 \gamma}$ (根据 $\varepsilon_{0}$ 的定义, 我们很容易得到 $\varepsilon=2^{-6 \gamma \theta}$, 其中 $\theta$ 是一个依赖于 $s$ 和 $\beta$ 的 正数). 令 $Q_{2}=\log _{2}\left(12 \gamma(\varepsilon)^{-1}\right)$, 那么当 $q>Q_{2}$ 时, 有

$$
\left\|T_{q+3 l} u\right\|_{L^{\infty}} \leqslant\left(2^{-6 \gamma}\right)^{l} A_{q}+2^{-\gamma(q+3 l)} A_{q}+C \cdot 2^{-\gamma(q+3 l)} .
$$

当 $l>q$ 时, 可以得到

$$
\left(2^{-6 \gamma l}\right)=2^{-\gamma(q+3 l)} 2^{\gamma(q-3 l)} \leqslant C \cdot 2^{-\gamma(q+3 l)} .
$$

最后, 如果选择 $Q_{0}=\max \left\{Q_{1}, Q_{2}\right\}$, 当 $q>Q_{0}$ 和 $l>q$ 时, 利用 (2.18) 可以得到

$$
\left\|T_{q+3 l} u\right\|_{L^{\infty}} \leqslant C \cdot 2^{-\gamma(q+3 l)} .
$$

因此, $u(t, x)$ 满足 $(2.5)$, 定理 2.1 证明完毕.

注 2.6 这里之所以将以上方法称为 “开放式高频估计”, 是因为其估计是针对解的高频部分, 并 且开放式地逐步向更高频进行迭代.

\section{3 解的 $L^{p}$ 范数下的有界性估计}

本节将得到方程解的 $L^{p}$ 范数下的有界性, 首先考虑方程解的 $L^{1}$ 范数下的有界性.

\section{$3.1 L^{1}$ 范数下的有界性}

首先给出下面的关于解在 $L^{1}$ 范数下的有界性的引理:

引理 3.1 假设 $\left\|u_{0}\right\|_{L^{1}} \leqslant C, u(t, x)$ 是方程 (1.1) 的解, 那么一定有

$$
\|u\|_{L^{1}} \leqslant\left\|u_{0}\right\|_{L^{1}} .
$$

类似的结论可参见文献 [10]. 为了方便读者, 这里给出简单的证明过程.

引理 3.1 的证明 设 $\varphi$ 为光滑子. 当 $\delta>0$ 时, 令 $\varphi_{\delta}(x)=\frac{1}{\delta} \varphi\left(\frac{x}{\delta}\right)$. 另外, 令 $\operatorname{sgn}$ 为定义在实数集上 的符号函数, 那么它的光滑子为 $\operatorname{sgn}_{\delta}=\varphi_{\delta} * \operatorname{sgn}(\delta>0)$. 在方程 $(1.1)$ 两端同时乘以 $\operatorname{sgn}_{\delta}(u)$ 并且在 $\mathbb{R}^{3}$ 上积分可得

$$
\frac{d}{d t} \int_{\mathbb{R}^{3}} \int_{0}^{u} \operatorname{sgn}_{\delta}(\eta) d \eta d x+\int_{\mathbb{R}^{3}} \operatorname{sgn}_{\delta}(u) \nabla \cdot f(u) d s+\int_{\mathbb{R}^{3}} \operatorname{sgn}_{\delta}(u) \Gamma_{s} u d x=0 .
$$

注意到

$$
\operatorname{sgn}_{\delta}(u) \nabla \cdot f(u)=\nabla \cdot\left[\operatorname{sgn}_{\delta}(u)(f(u)-f(0))\right]-\nabla \cdot\left[\int_{0}^{u} \operatorname{sgn}_{\delta}^{\prime}(\eta)(f(\eta)-f(0)) d \eta\right] .
$$

因此, (3.2) 左端的第二项为 0 , 即

$$
\frac{d}{d t} \int_{\mathbb{R}^{3}} \int_{0}^{u} \operatorname{sgn}_{\delta}(\eta) d \eta d x+\int_{\mathbb{R}^{3}} \operatorname{sgn}_{\delta}(u) \Gamma_{s} u d x=0 .
$$

把 (3.3) 关于时间 $t$ 在 $[0, t]$ 积分, 从而可以得到对于任意的 $0 \leqslant t \leqslant T$ 都有 (3.1). 最后, 令 $\delta \rightarrow 0$, 完 成了引理 3.1 的证明. 


\section{$3.2 L^{2}$ 范数下的衰减估计}

接下来将利用加权能量估计的方法得到方程解在 $L^{2}$ 范数下的衰减性. 我们的结论如下:

定理 3.2 假设 $\left\|u_{0}\right\|_{L^{1} \cap L^{2}} \leqslant C, u(t, x)$ 是方程 (1.1) 的解, 那么 $u(t, x)$ 有下面的衰减性:

$$
\|u(t)\|_{L^{2}} \leqslant C\left\|u_{0}\right\|_{L^{1} \cap L^{2}}(1+t)^{-\frac{3}{4}} .
$$

证明 首先给出 $u(t, x)$ 在 $L^{2}$ 范数下的有界性. 在方程 (1.1) 两端同时乘以 $2 u$, 积分可得

$$
\|u\|_{L^{2}}^{2}+2 \int_{0}^{t}\left\|\Lambda\langle\Lambda\rangle^{-\frac{s}{2}} u(\tau)\right\|_{L^{2}}^{2} d \tau=\left\|u_{0}\right\|_{L^{2}}^{2},
$$

其中 $\langle\Lambda\rangle$ 的 Fourier 变换为

$$
\mathcal{F}(\langle\Lambda\rangle)=\langle\xi\rangle:=\left(1+|\xi|^{2}\right)^{\frac{1}{2}} .
$$

因此, 我们得到了 $u(t, x)$ 在 $L^{2}$ 范数下的有界性.

接下来将利用加权能量估计的办法来得到方程解在 $L^{2}$ 范数下的衰减性. 在方程 (1.1) 两端同时 乘以 $2(1+t)^{a} u$ 并且在 $\mathbb{R}^{3}$ 积分可以得到

$$
\left\|(1+t)^{\frac{a}{2}} u\right\|_{L^{2}}^{2}+2 \int_{0}^{t}(1+\tau)^{a}\left\|\Lambda\langle\Lambda\rangle^{-\frac{s}{2}} u(\tau)\right\|_{L^{2}}^{2} d \tau=\left\|u_{0}\right\|_{L^{2}}^{2}+\int_{0}^{t} a(1+\tau)^{a-1}\|u\|_{L^{2}}^{2} d \tau .
$$

事实上, 由于 $0 \leqslant s \leqslant 1$, 我们很容易得到

$$
\|u\|_{L^{2}}^{2} \leqslant\left\|\langle\Lambda\rangle^{-\frac{s}{2}} u\right\|_{L^{2}}^{2}+\left\|\Lambda\langle\Lambda\rangle^{-\frac{s}{2}} u\right\|_{L^{2}}^{2} .
$$

利用 (3.6), 可以得到

$$
\left\|(1+t)^{\frac{a}{2}} u\right\|_{L^{2}}^{2}+2 \int_{0}^{t}(1+\tau)^{a}\left\|\Lambda\langle\Lambda\rangle^{-\frac{s}{2}} u(\tau)\right\|_{L^{2}}^{2} d \tau \leqslant\left\|u_{0}\right\|_{L^{2}}^{2}+N_{1}+N_{2},
$$

其中

$$
N_{1}=a \int_{0}^{t}(1+\tau)^{a-1}\left\|\Lambda\langle\Lambda\rangle^{-\frac{s}{2}} u(\tau)\right\|_{L^{2}}^{2} d \tau
$$

和

$$
N_{2}=a \int_{0}^{t}(1+\tau)^{a-1}\left\|\langle\Lambda\rangle^{-\frac{s}{2}} u(\tau)\right\|_{L^{2}}^{2} d \tau
$$

接下来将估计 $N_{1}$ 和 $N_{2}$ 这两项. 当 $a>\frac{3}{2}$ 时, 由于 $(a-1) / a+1 / a=1$, 利用 Young 不等式和 (3.5), 我们可以得到

$$
N_{1} \leqslant \eta \int_{0}^{t}(1+\tau)^{a}\left\|\Lambda\langle\Lambda\rangle^{-\frac{s}{2}} u(\tau)\right\|_{L^{2}}^{2} d \tau+C_{a, \eta}\left\|u_{0}\right\|_{L^{2}}^{2},
$$

其中 $\eta$ 为一个待定的正数. 为了估计 $N_{2}$, 我们有下面的不等式:

$$
\begin{aligned}
\int_{\mathbb{R}^{3}} \frac{1}{\left(1+|\xi|^{2}\right)^{s / 2}}|\hat{u}|^{2} d \xi & =\int_{|\xi|>R} \frac{1}{\left(1+|\xi|^{2}\right)^{s / 2}}|\hat{u}|^{2} d \xi+\int_{|\xi| \leqslant R} \frac{1}{\left(1+|\xi|^{2}\right)^{s / 2}}|\hat{u}|^{2} d \xi \\
& \leqslant \frac{1}{R^{2}} \int_{\mathbb{R}^{3}} \frac{|\xi|^{2}}{\left(1+|\xi|^{2}\right)^{s / 2}}|\hat{u}|^{2} d \xi+C\|\hat{u}\|_{L^{\infty}}^{2} R^{3}
\end{aligned}
$$


其中 $R$ 为任意大的正数. 特别地, 取 $R$ 的值为

$$
\frac{1}{R^{2}} \int_{\mathbb{R}^{3}} \frac{|\xi|^{2}}{\left(1+|\xi|^{2}\right)^{s / 2}}|\hat{u}|^{2} d \xi=C\|\hat{u}\|_{L^{\infty}}^{2} R^{3},
$$

也即

$$
R=\left(\frac{\int_{\mathbb{R}^{3}} \frac{|\xi|^{2}}{\left(1+|\xi|^{2}\right)^{s / 2}}|\hat{u}|^{2} d \xi}{C\|\hat{u}\|_{L^{\infty}}^{2}}\right)^{\frac{1}{5}}
$$

从而可以得到

$$
\int_{\mathbb{R}^{3}} \frac{1}{\left(1+|\xi|^{2}\right)^{s / 2}}|\hat{u}|^{2} d \xi \leqslant C\left(\int_{\mathbb{R}^{3}} \frac{|\xi|^{2}}{\left(1+|\xi|^{2}\right)^{s / 2}}|\hat{u}|^{2} d \xi\right)^{\frac{3}{5}}\|\hat{u}\|_{L^{\infty}}^{\frac{4}{5}} .
$$

利用 Young 不等式, 我们可以得到

$$
\begin{aligned}
N_{2} & =a \int_{0}^{t}(1+\tau)^{a-1}\left\|\langle\Lambda\rangle^{-\frac{s}{2}} u(\tau)\right\|_{L^{2}}^{2} d \tau \\
& \leqslant \eta \int_{0}^{t}(1+\tau)^{a} \int_{\mathbb{R}^{3}} \frac{|\xi|^{2}}{\left(1+|\xi|^{2}\right)^{s / 2}}|\hat{u}|^{2} d \xi d \tau+C_{k, \eta}\left\|u_{0}\right\|_{L^{1}}^{2}(1+t)^{a-\frac{3}{2}}
\end{aligned}
$$

将 (3.8) 和 (3.9) 代入到 (3.7), 并且取 $0<\eta<\frac{1}{2}$, 可得

$$
(1+t)^{a}\|u\|_{L^{2}}^{2}+(2-2 \eta) \int_{0}^{t}(1+\tau)^{a}\left\|\Lambda\langle\Lambda\rangle^{-\frac{s}{2}} u\right\|_{L^{2}}^{2} d \tau \leqslant C\left\|u_{0}\right\|_{L^{2}}^{2}+C\left\|u_{0}\right\|_{L^{1}}^{2}(1+t)^{a-\frac{3}{2}} .
$$

由于一开始选择 $a>\frac{3}{2}$, 从而得到了定理 3.2 的证明.

\section{$3.3 L^{p}(p>2)$ 范数下的估计}

在上面两小节中得到了方程解在 $L^{1}$ 和 $L^{2}$ 范数下的有界性. 关于 $\|u\|_{L^{p}}(2<p \leqslant \infty)$ 的有界性, 我们有下面的极大值原理:

定理 3.3 (极大值原理) 假设 $u_{0}(x) \in L^{1}\left(\mathbb{R}^{3}\right) \cap L^{2}\left(\mathbb{R}^{3}\right), u(t, x)$ 是方程 (1.1) 的解, 并且 $0 \leqslant s \leqslant 1$, 那么有

$$
\|u(t)\|_{L^{p}} \leqslant C\left\|u_{0}\right\|_{L^{p}}
$$

其中 $2<p \leqslant+\infty$.

为了给出定理 3.3 的证明, 我们需要算子 $\Lambda^{s}$ 的正性定理.

引理 3.4 设 $s \in[0,2], u(x) \in \mathcal{S}(\Omega)$, 其中 $\Omega=\mathbb{R}^{3}$, 则算子 $\Lambda^{s}$ 有下面的逐点估计:

$$
2 u(x) \Lambda^{s} u(x) \geqslant \Lambda^{s} u^{2}(x) .
$$

当 $\Omega=\mathbb{R}^{2}, \mathbb{T}^{2}$ 时, 文献 [35] 给出了详细的证明过程, 而上述引理的证明完全类似于文献 $[35,36]$ 的证明, 为了简洁起见, 我们不再给出证明. 为完成定理 3.3 的证明, 还需要关于 $\left\|\Lambda^{-s} u\right\|_{L^{p}}$ 的估计, 即 下面的引理 (证明参见文献 [30, 第 119 页定理 1$]$ ):

引理 3.5 若 $0<s<3,1 \leqslant p<q<\infty$, 而且满足 $1 / q+s / 3=1 / p$, 那么有

$$
\left\|\Lambda^{-s} f\right\|_{L^{q}} \leqslant C\|f\|_{L^{p}}
$$

600 
定理 3.3 的证明 根据 $\Gamma_{s}$ 和 $\Lambda^{s}$ 定义, 我们将方程 (1.1) 重新改写为

$$
\partial_{t} u+\Lambda^{2-s} u=\operatorname{div} f(u)+\left(\Lambda^{2-s}-\Gamma_{s}\right) u .
$$

在方程 (3.11) 两端同时乘以 $p u^{p-1}$ (其中 $p=2^{n}, n=2,3, \ldots$ ), 积分可得

$$
\frac{d}{d t}\|u\|_{L^{p}}^{p}+\left\|\Lambda^{1-\frac{s}{2}} u^{\frac{p}{2}}\right\|_{L^{2}}^{2}=p \int_{\mathbb{R}^{3}} u^{p-1}\left(\Lambda^{2-s}-\Gamma_{s}\right) u d x .
$$

接下来将利用高低频分解的办法来处理方程 (3.12) 的右端项. 令

$$
\chi(\xi)= \begin{cases}1, & |\xi|>2 R, \\ 0, & |\xi|<R\end{cases}
$$

为光滑的截断算子, $R$ 为一个大于 2 的正数. 算子 $\chi(D)$ 是一个以 $\chi(\xi)$ 为核的算子. 引入了算子 $\chi(D)$ 后, 方程 (3.12) 可以写为

$$
\frac{d}{d t}\|u\|_{L^{p}}^{p}+\left\|\Lambda^{1-\frac{s}{2}} u^{\frac{p}{2}}\right\|_{L^{2}}^{2}=: K_{1}+K_{2}
$$

其中 $K_{1}$ 和 $K_{2}$ 分别为

$$
K_{1}=\left|\int_{\mathbb{R}^{3}} u^{p-1}\left(\Lambda^{2-s}-\Gamma_{s}\right)_{L} u d x\right|, \quad K_{2}=\left|\int_{\mathbb{R}^{3}} u^{p-1}\left(\Lambda^{2-s}-\Gamma_{s}\right)_{H} u d x\right|,
$$

其中

$$
\begin{aligned}
& \left(\Lambda^{2-s}-\Gamma_{s}\right)_{L}=(I-\chi(D))\left(\Lambda^{2-s}-\Gamma_{s}\right), \\
& \left(\Lambda^{2-s}-\Gamma_{s}\right)_{H}=\chi(D)\left(\Lambda^{2-s}-\Gamma_{s}\right) .
\end{aligned}
$$

结合算子 $\Lambda^{2-s}-\Gamma_{s}$ 的具体性质, 我们有下面的估计:

$$
K_{1} \leqslant C\left\|\left(\Lambda^{2-s}-\Gamma_{s}\right)_{L} u\right\|_{L^{\infty}}\|u\|_{L^{p-1}}^{p-1} \leqslant C\|u\|_{L^{2}}\|u\|_{L^{p-1}}^{p-1} \leqslant C\|u\|_{L^{2}}^{\frac{p}{p-2}}\|u\|_{L^{p}}^{\frac{p(p-3)}{p-2}} .
$$

利用 Gagliardo-Nirenberg 不等式, 有

$$
\begin{aligned}
\|u\|_{L^{p}} & =\left\|u^{\frac{p}{2}}\right\|_{L^{2}}^{\frac{2}{p}} \leqslant C\left\|\Lambda^{1-\frac{s}{2}} u^{\frac{p}{2}}\right\|_{L^{2}}^{\frac{3}{5-3} \cdot \frac{2}{p}}\left\|u^{\frac{p}{2}}\right\|_{L^{1}}^{\frac{2-s}{5-s} \cdot \frac{2}{p}} \\
& \leqslant C\left\|\Lambda^{1-\frac{s}{2}} u^{\frac{p}{2}}\right\|_{L^{2}}^{\frac{6}{p(5-s)}}\left(\|u\|_{L^{2}}^{\beta}\|u\|_{L^{p}}^{1-\beta}\right)^{\frac{2-s}{5-s}} \\
& \leqslant C\left\|\Lambda^{1-\frac{s}{2}} u^{\frac{p}{2}}\right\|_{L^{2}}^{\frac{6}{p(5-s)}}\|u\|_{L^{2}}^{\frac{2}{p-2} \frac{2-s}{5-s}}\|u\|_{L^{p}}^{\frac{p-4}{p-2} \frac{2-s}{5-s}}
\end{aligned}
$$

从而,

$$
\|u\|_{L^{p}} \leqslant C\left\|\Lambda^{1-\frac{s}{2}} u^{\frac{p}{2}}\right\|_{L^{2}}^{\frac{6(p-2)}{p(3 p-2 s-2)}}\|u\|_{L^{2}}^{\frac{2(2-s)}{3 p-2 s-2}} .
$$

利用 (3.14) 和 Young 不等式, 可以得到

$$
K_{1} \leqslant C\left\|\Lambda^{1-\frac{s}{2}} u^{\frac{p}{2}}\right\|_{L^{2}}^{\frac{6(p-3)}{3 p-2 s-2}}\|u\|_{L^{2}}^{\frac{(7-2 s) p}{3 p-2 s-2}} \leqslant C \varepsilon\left\|\Lambda^{1-\frac{s}{2}} u^{\frac{p}{2}}\right\|_{L^{2}}^{2}+C\|u\|_{L^{2}}^{p} .
$$

最后来估计 $K_{2}$, 当 $|\xi|$ 充分大时,

$$
\sigma\left(\Lambda^{2-s}-\Gamma_{s}\right)=\frac{s}{2}|\xi|^{-s}+\frac{\frac{s}{2}\left(\frac{s}{2}+1\right)}{2 !}|\xi|^{-2-s}+O\left(|\xi|^{-2-s}\right) .
$$


将 $K_{2}$ 改写为

$$
K_{2} \leqslant\left|\int_{\mathbb{R}^{3}} u^{p-1}\left(\Lambda^{-s}\right)_{H} u d x\right|+\left|\int_{\mathbb{R}^{3}} u^{p-1}\left(\Lambda^{2-s}-\Gamma_{s}-\frac{s}{2} \Lambda^{-s}\right)_{H} u d x\right|=: K_{21}+K_{22} .
$$

对于 $K_{21}$, 利用 Cauchy 不等式, 有

$$
K_{21}=\left|\int_{\mathbb{R}^{3}} u^{p-1}\left(\Lambda^{-s}\right)_{H} u d x\right| \leqslant C \int_{\mathbb{R}^{3}}\left|u^{p-1} \Lambda^{-s} u\right| d x \leqslant C\|u\|_{L^{p}}^{p-1}\left\|\Lambda^{-s} u\right\|_{L^{p}} .
$$

由引理 3.5 可以得到

$$
K_{21} \leqslant\|u\|_{L^{p}}^{p-1}\|u\|_{L^{r}} \leqslant C\|u\|_{L^{p}}^{p-1}\|u\|_{L^{2}}^{\theta}\|u\|_{L^{p}}^{1-\theta},
$$

其中 $r=\frac{3 p}{3+s p}<p$ 并且 $\frac{1}{r}=\frac{\theta}{2}+\frac{1-\theta}{p}$. 另外, 由 (3.14) 可以得到

$$
\begin{aligned}
K_{21} & \leqslant C\|u\|_{L^{2}}^{\frac{2 s p}{3(p-2)}}\left[\left\|\Lambda^{1-\frac{s}{2}} u^{\frac{p}{2}}\right\|_{L^{2}}^{\frac{6(p-2)}{p(3 p-2 s-2)}}\|u\|_{L^{2}}^{\frac{2(2-s)}{3 p-2 s-2}}\right]^{\frac{3 p(p-2)-2 s p}{3(p-2)}} \\
& \leqslant C\left\|\Lambda^{1-\frac{s}{2}} u^{\frac{p}{2}}\right\|_{L^{2}}^{\frac{2(3 p-2 s-6)}{3 p-2 s-2}}\|u\|_{L^{2}-2 s-2}^{\frac{4 p}{3 p-2}} \leqslant \varepsilon\left\|\Lambda^{1-\frac{s}{2}} u^{\frac{p}{2}}\right\|_{L^{2}}^{2}+C\|u\|_{L^{2}}^{p} .
\end{aligned}
$$

为了给出 $K_{22}$ 的估计, 我们先给出 $\left\|\left(\Lambda^{2-s}-\frac{s}{2} \Lambda^{-s}-\Gamma_{s}\right)_{H} u\right\|_{L^{\infty}}$ 的估计,

$$
\left\|\left(\Lambda^{2-s}-\frac{s}{2} \Lambda^{-s}-\Gamma_{s}\right)_{H} u\right\|_{L^{\infty}} \leqslant C\left(\int_{|\xi| \geqslant R}|\xi|^{-4-2 s} d \xi\right)^{\frac{1}{2}}\left(\int_{|\xi| \geqslant R}|\hat{u}|^{2} d \xi\right)^{\frac{1}{2}} \leqslant C\|u\|_{L^{2}} .
$$

最后, 类似于 $K_{1}$ 的估计方法, 我们可以得到

$$
K_{22} \leqslant \varepsilon\left\|\Lambda^{1-\frac{s}{2}} u^{\frac{p}{2}}\right\|_{L^{2}}^{2}+C\|u\|_{L^{2}}^{p} .
$$

将 $K_{1} 、 K_{21}$ 和 $K_{22}$ 的估计结合在一起, 可以得到

$$
\frac{d}{d t}\|u\|_{L^{p}}^{p}+\left\|\Lambda^{1-\frac{s}{2}} u^{\frac{p}{2}}\right\|_{L^{2}}^{2} \leqslant 3 \varepsilon\left\|\Lambda^{1-\frac{s}{2}} u^{\frac{p}{2}}\right\|_{L^{2}}^{2}+C\|u\|_{L^{2}}^{p} .
$$

取 $0<\varepsilon \leqslant \frac{1}{4}$, 注意到我们有 $\|u\|_{L^{2}} \leqslant C(1+t)^{-\frac{3}{4}}$, 则可以得到

$$
\|u\|_{L^{p}}^{p}+\frac{1}{4} \int_{0}^{t}\left\|\Lambda^{1-\frac{s}{2}} u^{\frac{p}{2}}\right\|_{L^{2}}^{2} d \tau \leqslant C,
$$

其中 $C$ 是一个依赖于 $\left\|u_{0}\right\|_{L^{1}}$ 和 $\left\|u_{0}\right\|_{L^{2}}$ 的常数. 证毕.

\section{4 当 $s=1$ 时, $C^{\alpha}$ 范数下的有界性估计}

对次临界的情形, 即当 $0 \leqslant s<1$ 时, 第 3 节已经证明了方程的解是满足正则性准则的条件, 即 $\|u\|_{L^{p}}<+\infty$. 接下来将证明当 $s=1$ 时, 方程的解也是满足正则性准则的条件, 即 $u(t, x) \in C^{\alpha}$.

当 $s=1$ 时, 我们所研究的方程为

$$
\begin{cases}\partial_{t} u-\frac{\Delta}{(1-\Delta)^{\frac{1}{2}}} u=\operatorname{div} f(u), & x \in \mathbb{R}^{3}, \quad t>0, \\ u(x, 0)=u_{0}(x), & x \in \mathbb{R}^{3} .\end{cases}
$$

我们的结论如下: 
定理 4.1 假设 $u_{0}$ 属于 $C^{\alpha}, u(t, x)$ 是 Cauchy 问题 (4.1) 的解, 则对充分小的正数 $\alpha$, 有 $u \in C^{\alpha}$.

这个定理的证明需要用到 Constantin 等 [24] 得到的非线性极大值原理. 首先我们强调一下, 在后 面的证明中, 我们假设方程的解 $u(t, x)$ 是一个光滑函数. 如其不然, 我们可以在方程左端增加一个人 为黏性项, 如 $-\epsilon \triangle u$. 同时注意到下面的所有证明的有界性均是不依赖于 $\epsilon$ 的, 于是再令 $\epsilon \rightarrow 0$ 就可 以得到结果.

\section{定理 4.1 的证明 令}

$$
v=\frac{\delta_{h} u(x)}{|h|^{\alpha}}
$$

其中 $\delta_{h} u=u(x+h, t)-u(x, t)$, 那么,

$$
\left(\partial_{t}+u \sum_{j=1}^{3} \partial_{x_{j}}+\left(\delta_{h} u\right) \sum_{j=1}^{3} \partial_{h_{j}}+\Lambda\right)\left(\delta_{h} u\right)=-\left(\Gamma_{1}-\Lambda\right)\left(\delta_{h} u\right) .
$$

上述方程两端同时乘以 $\frac{\delta_{h} u}{|h|^{2 \alpha}}$, 则得到下面的方程:

$$
\frac{1}{2}\left(\partial_{t}+u \sum_{j=1}^{3} \partial_{x_{j}}+\left(\delta_{h} u\right) \sum_{j=1}^{3} \partial_{h_{j}}+\Lambda\right) v^{2}+\frac{D\left[\delta_{h} u\right]}{2|h|^{2 \alpha}}=\frac{\alpha}{|h|^{2 \alpha+1}}\left(\delta_{h} u\right)^{3}-\frac{\delta_{h} u}{|h|^{2 \alpha}}\left(\Gamma_{1}-\Lambda\right)\left(\delta_{h} u\right) .
$$

上面用到了文献 [35] 中关于 $\Lambda$ 算子的逐点估计，

$$
2 \varphi(x) \Lambda \varphi(x)=\Lambda\left(\varphi^{2}(x)\right)+D[\varphi(x)],
$$

其中 $D[\varphi(x)]$ 为

$$
D[\varphi(x)]=\mathrm{P} . \mathrm{V} \cdot \int_{R^{3}}(\varphi(x)-\varphi(x+y))^{2} \frac{c}{|y|^{d+\alpha}} d y .
$$

利用文献 [24] 中提到的方法, 可以得到

$$
D\left[\delta_{h} u\right] \geqslant \frac{\left|\delta_{h} u\right|^{3}}{C\|u\|_{L^{\infty}}|h|}
$$

因此,

$$
\begin{aligned}
& \left(\partial_{t}+u \sum_{j=1}^{3} \partial_{x_{j}}+\left(\delta_{h} u\right) \sum_{j=1}^{3} \partial_{h_{j}}+\Lambda\right) v^{2}+\frac{\left|\delta_{h} u\right|^{3}}{C\|u\|_{L^{\infty}}|h|^{2 \alpha+1}} \\
& \quad \leqslant \frac{2 \alpha}{|h|^{2 \alpha+1}}\left(\delta_{h} u\right)^{3}-2 \frac{\delta_{h} u}{|h|^{2 \alpha}}\left(\Gamma_{1}-\Lambda\right)\left(\delta_{h} u\right) .
\end{aligned}
$$

如果 $\alpha$ 充分小, 那么,

$$
\left(\partial_{t}+u \sum_{j=1}^{3} \partial_{x_{j}}+\left(\delta_{h} u\right) \sum_{j=1}^{3} \partial_{h_{j}}+\Lambda\right) v^{2}+\frac{\left|\delta_{h} u\right|^{3}}{2 C\|u\|_{L^{\infty}}|h|^{2 \alpha+1}} \leqslant 2 \frac{\left|\delta_{h} u\right|}{|h|^{2 \alpha}}\left\|\left(\Gamma_{1}-\Lambda\right)\left(\delta_{h} u\right)\right\|_{L^{\infty}} .
$$

现在估计上述不等式的右端项, 经过直接计算可知,

$$
\left\|\left(\Gamma_{1}-\Lambda\right)\left(\delta_{h} u\right)\right\|_{L^{\infty}} \leqslant\left\|\left(\Gamma_{1}-\Lambda+\frac{1}{2}\langle\Lambda\rangle^{-1}\right)\left(\delta_{h} u\right)\right\|_{L^{\infty}}+\frac{1}{2}\left\|\langle\Lambda\rangle^{-1}\left(\delta_{h} u\right)\right\|_{L^{\infty}}
$$




$$
\begin{aligned}
& \leqslant\left\|\mathcal{F}\left(\left(\Gamma_{1}-\Lambda+\frac{1}{2}\langle\Lambda\rangle^{-1}\right)\left(\delta_{h} u\right)\right)\right\|_{L^{1}}+\frac{1}{2}\left\|\mathcal{K}_{1} *\left(\delta_{h} u\right)\right\|_{L^{\infty}} \\
& \leqslant\left\|\left[\frac{|\xi|^{2}}{\left(1+|\xi|^{2}\right)^{\frac{1}{2}}}-|\xi|+\frac{1}{2} \frac{1}{\left(1+|\xi|^{2}\right)^{\frac{1}{2}}}\right] \mathcal{F}\left(\delta_{h} u\right)\right\|_{L^{1}}+\frac{1}{2}\left\|\mathcal{K}_{1}\right\|_{L^{1}}\left\|\left(\delta_{h} u\right)\right\|_{L^{\infty}},
\end{aligned}
$$

其中 $\mathcal{K}_{1}(x)$ 是算子 $\langle\Lambda\rangle^{-1}=(I-\Delta)^{-\frac{1}{2}}$ 的核, 由 $\mathcal{K}_{s}(x)$ 的定义 (见 (1.10)) 可知,

$$
\left\|\left(\Gamma_{1}-\Lambda\right)\left(\delta_{h} u\right)\right\|_{L^{\infty}} \leqslant M\left\|\left(\delta_{h} u\right)\right\|_{L^{2}}+\frac{1}{2}\left\|\left(\delta_{h} u\right)\right\|_{L^{\infty}},
$$

其中

$$
M=\left\|\frac{|\xi|^{2}}{\left(1+|\xi|^{2}\right)^{\frac{1}{2}}}-|\xi|+\frac{1}{2} \frac{1}{\left(1+|\xi|^{2}\right)^{\frac{1}{2}}}\right\|_{L^{2}},
$$

很容易看到, 上述积分的唯一的奇点是 $|\xi|=\infty$. 因此, 当 $|\xi|$ 充分大时, 利用 Taylor 展开, 可得

$$
\begin{aligned}
\frac{|\xi|^{2}}{\left(1+|\xi|^{2}\right)^{\frac{1}{2}}}-|\xi|+\frac{1}{2} \frac{1}{\left(1+|\xi|^{2}\right)^{\frac{1}{2}}} & =\frac{|\xi|^{2}}{\left(1+|\xi|^{2}\right)^{\frac{1}{2}}}\left[1-\left(1+\frac{1}{|\xi|^{2}}\right)^{\frac{1}{2}}+\frac{1}{2|\xi|^{2}}\right] \\
& =\frac{|\xi|^{2}}{\left(1+|\xi|^{2}\right)^{\frac{1}{2}}}\left[1-\left(1+\frac{1}{2} \frac{1}{|\xi|^{2}}-\frac{1}{4|\xi|^{4}}+O\left(\frac{1}{|\xi|^{4}}\right)\right)+\frac{1}{2|\xi|^{2}}\right] \\
& =O\left(\frac{1}{|\xi|^{3}}\right) .
\end{aligned}
$$

显然, 上面函数的平方是可积的, 于是 $M$ 是有界的. 也就有

$$
\left\|\left(\Gamma_{1}-\Lambda\right)\left(\delta_{h} u\right)\right\|_{L^{\infty}} \leqslant 2 M\|u\|_{L^{2}}+\|u\|_{L^{\infty}} \leqslant C_{0}
$$

综合以上讨论可知,

$$
2 \frac{\left|\delta_{h} u\right|}{|h|^{2 \alpha}}\left\|\left(\Gamma_{1}-\Lambda\right)\left(\delta_{h} u\right)\right\|_{L^{\infty}} \leqslant 2 C_{0} \frac{\left|\delta_{h} u\right|}{|h|^{2 \alpha}} \leqslant\left(\frac{\left|\delta_{h} u\right|}{\left(4 C\|u\|_{L^{\infty}}\right)^{\frac{1}{3}}|h|^{\frac{2 \alpha+1}{3}}}\right)^{3}+C\left(\frac{1}{|h|^{2 \alpha-\frac{2 \alpha+1}{3}}}\right)^{\frac{3}{2}} .
$$

从而有

$$
\left(\partial_{t}+u \sum_{j=1}^{3} \partial_{x_{j}}+\left(\delta_{h} u\right) \sum_{j=1}^{3} \partial_{h_{j}}+\Lambda\right) v^{2}+\frac{\left|\delta_{h} u\right|^{3}}{4 C\|u\|_{L^{\infty}}|h|^{2 \alpha+1}} \leqslant C|h|^{\frac{1}{2}-2 \alpha} .
$$

现在仅需要考虑的是 $|h| \leqslant 1$ 的情形. 事实上, 对 $|h|>1$ 的情形, 我们有

$$
\|v\|_{L_{x, h}^{\infty}}=\left\|\frac{\left|\delta_{h} u(x)\right|}{|h|^{\alpha}}\right\|_{L_{x, h}^{\infty}} \leqslant C\|u\|_{L^{\infty}} \leqslant C .
$$

当 $|h| \leqslant 1$ 时, 由 $(4.8)$ 可以得到

$$
\left(\partial_{t}+u \sum_{j=1}^{3} \partial_{x_{j}}+\left(\delta_{h} u\right) \sum_{j=1}^{3} \partial_{h_{j}}+\Lambda\right) v^{2}+\frac{\left|\delta_{h} u\right|^{3}}{4 C\|u\|_{L^{\infty}}|h|^{2 \alpha+1}} \leqslant C .
$$

假设 $(\bar{x}, \bar{h})$ 是 $v^{2}$ 的极大值点, 那么,

$$
\partial_{x_{j}} v^{2}(\bar{x}, t ; \bar{h})=\partial_{h_{j}} v^{2}(\bar{x}, t ; \bar{h})=0, \quad \Lambda v^{2}(\bar{x}, t ; \bar{h}) \geqslant 0,
$$

604 
这里用到了算子 $\Lambda$ 的积分形式,

$$
\Lambda f(x)=C \mathrm{P} . \mathrm{V} \cdot \int_{\mathbb{R}^{3}} \frac{f(x)-f(x-y)}{|y|^{4}} d y .
$$

因此,

$$
\left(\partial_{t} v^{2}\right)(\bar{x}, t ; \bar{h})+C v^{3}(\bar{x}, t ; \bar{h}) \leqslant C
$$

用常微分方程的知识, 类似于文献 [23] 中定理 4.3 的证明, 我们可以得到

$$
\|u\|_{C^{\alpha}} \leqslant\|v\|_{L_{x, h}^{\infty}} \leqslant \tilde{C}
$$

其中 $\tilde{C}$ 是依赖于 $\left\|u_{0}\right\|_{C^{\alpha}}$ 和 $\left\|u_{0}\right\|_{L^{1}}$ 的常数. 证毕.

\section{参考文献}

1 Smoller J. Shock Waves and Reaction-Diffusion Equations. New York: Springer, 2012

2 Dafermos C M. Hyperbolic Conservation Laws in Continuum Physics, 2nd ed. Grundlehren der Mathematischen Wissenschaften, vol. 325. Berlin: Springer-Verlag, 2005

3 Goodman J. Nonlinear asymptotic stability of viscous shock profiles for conservation laws. Arch Ration Mech Anal, 1986, 95: 325-344

4 Hopf E. The partial differential equation $u_{t}+u u_{x}=\mu u_{x x}$. Comm Pure Appl Math, 1950, 3: 201-230

5 Kotlow D B. Quasilinear parabolic equations and first order quasilinear conservation laws with bad Cauchy data. J Math Anal Appl, 1971, 35: 563-576

6 Li F B, Wang W K. The pointwise estimates of solutions to the parabolic consevation law in multi-dimensions. NoDEA Nonlinear Differential Equations Appl, 2014, 21: 87-103

7 Schonbek M E. Decay of solution to parabolic conservation laws. Comm Partial Differential Equations, 1980, 5: 449-473

8 Schonbek M E. Uniform decay rates for parabolic conservation laws. Nonlinear Anal, 1986, 10: 943-956

9 Rosenau P. Extending hydrodynamics via the regularization of the Chapman-Enskog expansion. Phys Rev A (3), 1989, 40: 7193-7196

10 Duan R, Fellner K, Zhu C. Energy method for multi-dimensional balance laws with non-local dissipation. J Math Pures Appl (9), 2010, 93: 572-598

11 Gao W, Zhu C. Asymptotic decay toward the planar rarefaction waves for a model system of the radiating gas in two dimensions. Math Models Methods Appl Sci, 2008, 18: 511-541

12 Hamer K. Non-linear effects on the propagation of sound waves in a radiating gas. Quart J Mech Appl Math, 1971, 24: $155-168$

13 Kawashima S, Nishibata S. Cauchy problem for a model system of the radiating gas: Weak solutions with a jump and classical solutions. Math Models Methods Appl Sci, 1999, 9: 69-91

14 Liu Y, Kawashima S. Asymptotic behavior of solutions to a model system of a radiating gas. Commun Pure Appl Anal, 2011, 10: 209-223

15 Wang W K, Wang W J. The pointwise estimates of solutions for a model system of the radiating gas in multi-dimensions. Nonlinear Anal, 2009, 71: 1180-1195

16 Wang W J, Wang W K. Blow up and global existence of solutions for a model system of the radiating gas. Nonlinear Anal, 2013, 81: 12-30

17 Constantin P, Majda A J, Tabak E. Formation of strong fronts in the 2-d quasigeostrophic thermal active scalar. Nonlinearity, 1994, 7: 1495-1533

18 Held I M, Pierrehumbert R T, Garner S T, et al. Surface quasi-geostrophic dynamics. J Fluid Mech, 1995, 282: 1-20

19 Constantin P, Wu J. Behavior of solutions of 2D quasi-geostrophic equations. SIAM J Math Anal, 1999, 30: 937-948 
20 Schonbek M E, Schonbek T P. Asymptotic behavior to dissipative quasi-geostrophic flows. SIAM J Math Anal, 2003, 35: $357-375$

21 Wu J. Dissipative quasi-geostrophic equations with $L^{p}$ data. Electron J Differential Equations, 2001, 56: 1-13

22 Caffarelli L A, Vasseur A. Drift diffusion equations with fractional diffusion and the quasigeostrophic equation. Ann of Math (2), 2010, 171: 1903-1930

23 Constantin P, Tarfulea A, Vicol V. Long time dynamics of forced critical SQG. Comm Math Phys, 2015, 335: 93-141

24 Constantin P, Vicol V. Nonlinear maximum principles for dissipative linear nonlocal operators and applications. Geom Funct Anal, 2012, 22: 1289-1321

25 Kiselev A, Nazarov F, Volberg A. Global well-posedness for the critical 2D dissipative quasi-geostrophic equation. Invent Math, 2007, 167: 445-453

26 Chan C, Czubak M, Silvestre L. Eventual regularization of the slightly supercritical fractional Burgers equation. Discrete Contin Dyn Syst, 2010, 27: 847-861

27 Chan C, Czubak M. Regularity of solutions for the critical $N$-dimensional Burgers's equation. Ann Inst H Poincaré Anal Non Linéaire, 2010, 27: 471-501

28 Dong H, Du D, Li D. Finite time singularities and global well-posedness for fractal Burgers equations. Indiana Univ Math J, 2009, 58: 807-821

29 Kiselev A, Nazarov F, Shterenberg R. Blow-up and regularity for fractal Burgers equations. Dyn Partial Differ Equ, 2008, 5: 211-240

30 Stein E M. Singular Integrals and Differentiability Properties of Functions. Princeton: Princeton University Press, 2010

31 Bahouri H, Chemin J Y, Danchin R. Fourier Analysis and Nonlinear Partial Differential Equations. New York: Springer, 2011

32 Kato T. The Cauchy problem for quasi-linear symmetric hyperbolic systems. Arch Ration Mech Anal, 1975, 58: $181-205$

33 Liu T, Wang W. The pointwise estimates of diffusion wave for the Navier-Stokes systems in odd multi-dimensions. Comm Math Phys, 1998, 196: 145-173

34 Wang W, Yang T. The pointwise estimates of solutions for euler equations with damping in multi-dimensions. J Differential Equations, 2001, 173: 410-450

35 Córdoba A, Córdoba D. A maximum principle applied to quasi-geostrophic equations. Comm Math Phys, 2004, 249: $511-528$

$36 \mathrm{Ju} \mathrm{N}$. The maximum principle and the global attractor for the dissipative 2D quasi-geostrophic equations. Comm Math Phys, 2005, 255: 161-181

\section{附录 A 定理 2.4 的证明}

为了得到 Green 函数 $G(t, x)$ 的逐点估计, 首先将 Green 函数分成两部分, 低频部分 $G_{1}(t, x)$ 和高 频部分 $G_{2}(t, x)$, 其中 $G_{i}(t, x)=\chi_{i}(D) G(t, x)(i=1,2)$. 算子 $\chi_{i}(D)$ 的象征 $\chi_{i}(\xi)$ 是光滑的截断函数, 定义如下:

$$
\chi_{1}(\xi)=\left\{\begin{array}{ll}
1, & |\xi|<\epsilon, \\
0, & |\xi|>2 \epsilon,
\end{array} \quad \chi_{2}(\xi)=1-\chi_{1}(\xi)\right.
$$

其中 $0<\epsilon<1$.

首先给出一个非常重要的引理, 它在证明 Green 函数的逐点估计的过程中起到了关键的作用.

引理 A.1 (1) 假设 $\hat{f}(t, \xi)$ 是一个在 $\mathbb{R}^{n}$ 空间中具有紧支集的函数, 并且存在常数 $\nu>0$, 使得 $\hat{f}(t, \xi)$ 对于任意的正数 $k$ 和 $m$, 以及任意的复指标 $\alpha$ 和 $\beta,|\beta| \leqslant N$, 都有下面的不等式:

$$
\left|D_{\xi}^{\beta}\left(\xi^{\alpha} \hat{f}(\xi, t)\right)\right| \leqslant C|\xi|^{(|\alpha|+k-|\beta|)+}\left(1+\left(t|\xi|^{2}\right)\right)^{m} \mathrm{e}^{-\nu|\xi|^{2} t},
$$


那么一定有

$$
\left|D_{x}^{\alpha} f(t, x)\right| \leqslant C_{N} t^{-\frac{n+|\alpha|+k}{2}} B_{N}(t, x),
$$

其中 $N$ 为任意固定的正数, $(a)_{+}=\max (0, a)$,

$$
B_{N}(t, x)=\left(1+|x|^{2}(1+t)^{-1}\right)^{-N} .
$$

(2) 如果对于任意的 $t \geqslant 0, \operatorname{supp} \hat{f}(\cdot, \xi) \subset O_{\epsilon}=:\{\xi,|\xi|>\epsilon\}$ 都存在常数 $\nu>0$, 使得对于任意的正 数 $k$ 和 $m$ 以及复指标 $\alpha$ 和 $\beta,|\beta| \leqslant N$, 都有

$$
\left|D^{\beta}\left(\xi^{\alpha} \hat{f}(\xi, t)\right)\right| \leqslant C|\xi|^{|\alpha|+k-|\beta|}\left(1+\left(t|\xi|^{2-s}\right)\right)^{m} \mathrm{e}^{-\nu|\xi|^{2-s} t},
$$

那么一定有

$$
\left|D^{\alpha} f(t, x)\right| \leqslant C_{N} \mathrm{e}^{-\epsilon t / 2} t^{-\frac{n+|\alpha|+k}{2-s}} B_{N, s}(t, x),
$$

其中

$$
B_{N, s}(t, x)=\left(1+|x|^{2-s}(1+t)^{-1}\right)^{-N} .
$$

上述引理第一部分的证明参见文献 [34, 引理 A.1], 第二部分的证明与第一部分类似, 我们这里不 再赘述.

接下来将给出 Green 函数逐点估计的证明, 首先从低频部分开始, 关于低频部分的估计, 我们有 下面的结论:

引理 $\mathbf{A . 2}$ 对于任意的正整数 $N$, 如果 $\epsilon$ 充分小, 则一定存在常数 $C>0$, 使得

$$
\left|D_{x}^{\alpha} G_{1}\right| \leqslant C(1+t)^{-\frac{n+|\alpha|}{2}} B_{N}(t, x) .
$$

证明 当 $|\xi|$ 充分小时, 根据 $\hat{G}_{1}$ 表达形式, 经过计算可以得到

$$
\left|D_{\xi}^{\beta}\left(\xi^{\alpha} \hat{G}_{1}\right)\right| \leqslant C\left(|\xi|^{(|\alpha|-|\beta|)_{+}}+|\xi|^{|\alpha|} t^{\frac{|\beta|}{2}}\right)\left(1+t|\xi|^{2}\right)^{\frac{|\beta|}{2}+1} \mathrm{e}^{-|\xi|^{2} t} .
$$

由上面提到的引理 A.1 可得

$$
\left|D_{x}^{\alpha} G_{1}(t, x)\right| \leqslant C t^{-\frac{n+|\alpha|}{2}} B_{N}(t, x)
$$

并且,

$$
\left|D_{x}^{\alpha} G_{1}(t, x)\right| \leqslant C\left|\int_{\mathbb{R}^{3}} \mathrm{e}^{\sqrt{-1} x \cdot \xi} \chi_{1}(\xi)\left(\xi^{\alpha} \widehat{G}_{1}(t, \xi)\right) d \xi\right| \leqslant C\left|\int \chi_{1}(\xi) d \xi\right| \leqslant C .
$$

从而, 由 (A.2) 和 (A.3) 可以得到 (A.1), 也就是得到了 Green 函数低频部分的逐点估计.

当 $|\xi| \geqslant \epsilon$ 时, Green 函数的高频部分 $\widehat{G}_{2}(t, \xi)$ 有下列的估计:

$$
\left|D_{\xi}^{\beta}\left(\xi^{\alpha} \widehat{G}_{2}\right)\right| \leqslant C\left(|\xi|^{|\alpha|-|\beta|}\right)\left(1+|\xi|^{2-s} t\right)^{\frac{|\beta|}{2}+1} \mathrm{e}^{-|\xi|^{2-s} t} .
$$

再次利用引理 A.1(2), 可以得到下面的引理:

引理 A.3 若 $|\xi|>\epsilon$, 则

$$
\left|D_{x}^{\alpha} G_{2}(x, t)\right| \leqslant C t^{-\frac{n+|\alpha|}{2-s}} \mathrm{e}^{-\epsilon t / 2} B_{N, s}(x, t) .
$$


最后将高低频估计合在一起, 也就是把引理 A.2 和 A.3 结合在一起, 就可以得到 Green 函数的逐 点估计:

定理 A.4 对于任意的 $x \in \mathbb{R}^{n}$, Green 函数有下面的逐点估计:

$$
\begin{aligned}
& \left|D_{x}^{\alpha} G(x, t)\right| \leqslant C_{\alpha} t^{-\frac{n+|\alpha|}{2-s}} B_{N, s}(t, x), \quad 0<t<1, \\
& \left|D_{x}^{\alpha} G(x, t)\right| \leqslant C_{\alpha}(1+t)^{-\frac{n+|\alpha|}{2}} B_{N}(t, x), \quad t>1,
\end{aligned}
$$

而且,

$$
\begin{aligned}
& \left\|D^{\alpha} G(t, x)\right\|_{L^{p}} \leqslant C t^{-\frac{|\alpha|}{2-s}-\frac{n}{2-s}\left(1-\frac{1}{p}\right)}, \quad 1 \leqslant p \leqslant \infty, \quad 0<t<1, \\
& \left\|D^{\alpha} G(t, x)\right\|_{L^{p}} \leqslant C(1+t)^{-\frac{|\alpha|}{2}-\frac{n}{2}\left(1-\frac{1}{p}\right)}, \quad 1 \leqslant p \leqslant \infty, \quad t>1 .
\end{aligned}
$$

从而, 我们完成了定理 2.4 的证明.

\section{Global existence of large solutions to conservation laws with nonlocal dissipation-type terms}

Lijuan Wang, Weike Wang \& Xin Xu

Abstract We consider the global existence for large perturbation solutions of scalar conservation laws with nonlocal dissipative structures. At the very beginning, based on the Green function method and the LittlewoodPaley decomposition, we establish a new regularity criterion to improve the regularity of the solution. It is worth to point out that the key points in the regularity criterion are that $\|u\|_{L^{\infty}}<+\infty$ for $0 \leqslant s<1$ and $\|u\|_{C^{\alpha}}<+\infty$ for $s=1$. Thus, in the following part we devote ourselves to verify the two key points. For the subcritical case, we obtain the boundedness of solution in $L^{p}$ for any $p \in[2,+\infty)$ by the maximum principle. Thanks to the nonlinear maximum principle, $C^{\alpha}$ boundedness is established for the critical case. Finally, the global existence of classical solutions to the scalar conservation with large initial data is obtained.

Keywords scalar conservation law, dissipation, Cauchy problem, maximum principle, nonlinear maximum principle

$\operatorname{MSC}(2010) \quad 35 \mathrm{~S} 05,35 \mathrm{~S} 10,35 \mathrm{~B} 40$

doi: 10.1360/N012017-00138 